\title{
Bayesian posterior prediction of the patchy spatial distributions of small pelagic fish in regions of suitable habitat
}

\author{
Boyd Charlotte ${ }^{1,{ }^{*}}$, Woillez Mathieu ${ }^{1}$, Bertrand Sophie ${ }^{2}$, Castillo Ramiro ${ }^{3}$, Bertrand Arnaud ${ }^{2}$, \\ Punt André E ${ }^{1}$
}

${ }^{1}$ School of Aquatic and Fishery Sciences, University of Washington, Seattle, WA, USA 98105

2 IRD, UMR212 EME IFREMER/IRD/UM2, Avenue Jean 7 Monnet, BP 171, 34203 Sète Cedex, France

${ }^{3}$ Instituto del Mar del Perú, Esquina Gamarra y Gral. Valle s/n, Apartado 22, Callao, Lima, Peru

*Corresponding author : Charlotte Boyd, email address : boydchar@u.washington.edu

\begin{abstract}
:
Small pelagic fish aggregate within areas of suitable habitat to form patchy distributions with localized peaks in abundance. This presents challenges for geostatistical methods designed to investigate the processes underpinning the spatial distribution of stocks and simulate distributions for further analysis. In two-stage models, presence/absence is treated as separable and independent from the process explaining non-zero densities. This is appropriate where gaps in the distribution are attributable to one process and conditional abundance to another, but less so where patchiness is attributable primarily to the strong schooling tendencies of small pelagic fish within suitable habitat. We therefore developed a new modelling framework based on a truncated Gaussian Random Field (GRF) within a Bayesian framework. We evaluated this method using simulated test data, and then applied it to acoustic survey data for Peruvian anchoveta (Engraulis ringens). We assessed the method's performance in terms of posterior densities of spatial parameters, and the density distribution, spatial pattern, and overall spatial distribution of posterior predictions. We conclude that Bayesian posterior prediction based on a truncated GRF is effective at reproducing the patchiness of the observed spatial distribution of anchoveta.
\end{abstract}




\section{Introduction}

Acoustic surveys of small pelagic fish provide valuable information on their abundance and distribution (Simmonds and MacLennan 2005). The distribution of small pelagic fish typically can exhibit strong spatial structure (Fréon et al. 2005). A geostatistical approach is therefore ideal for inference and prediction because it takes into account spatial autocorrelation in the variance of the observed variable (Rivoirard et al. 2000). Geostatistical analysis may serve three broad purposes in fisheries ecology. First, structural analysis and inference may be used to investigate the relationship between the spatial distribution of stocks and environmental covariates, and the inherent spatial structure of fish stocks (Ciannelli et al. 2008, Lezama-Ochoa et al. 2011). Second, geostatistical methods may be used to estimate regional stock abundance (Rivoirard et al. 2000, Roa-Ureta and Niklitschek 2007). In recent years, conditional geostatistical simulation has been recognized as a useful tool for generating multiple spatial realisations of a variable and thus for estimating stock abundance given various sources of uncertainty (Gimona and Fernandes 2003, Walline 2007, Woillez et al. 2009). Third, conditional geostatistical simulation may be used to generate realisations of the spatial distribution of acoustic biomass over the survey region as a basis for analysing spatial associations, for instance between fishing fleets and fish stocks (see Bertrand et al. 2004b). The purpose of the geostatistical analysis presented here was to simulate the spatial distribution of small pelagic fish. The simulated spatial distribution was then used to analyse the foraging patterns of their predators (Boyd 2012). Nevertheless, we propose that the method described below is also of relevance to structural analysis and estimation of stock abundance and associated uncertainty.

The distribution of small pelagic fish is often characterized by intense patchiness (Petitgas 1993, Rivoirard et al. 2000), including gaps or areas of very low densities in the distribution. This leads to data with a high proportion of zeroes, and presents challenges for modelling (Ciannelli et al. 2008). The appropriate model structure for analysing these zeroes depends on the processes that underpin gaps in distribution. Gaps may be attributable to the association of the species with specific water masses or 
53 suitable habitat at the stock scale and/or to intrinsic aggregative behaviours leading to the formation of 54 schools and clusters of schools within suitable habitat (Fréon et al. 2005, Bertrand et al. 2008).

55 Where gaps in the distribution of small pelagic fish are attributable to areas of unsuitable habitat within a 56 survey region, the distribution of suitable habitat and fish densities within suitable habitat may be 57 modelled as separate processes. The conventional approach to modelling data with a high proportion of 58 zeroes is consistent with this scenario. A two-stage model structure may be developed, in which 59 presence/absence is treated as separable and independent from the process explaining non-zero densities 60 (Maunder and Punt 2004). For example, Walline (2007) applied three simulation methods to acoustic data 61 for walleye pollock (Theragra chalcogramma), including a two-stage model based on binary indicator 62 simulation for presence/absence; Roa-Ureta and Niklitschek (2007) analysed repeated trawl survey data 63 for squid (Loligo gahi) based on a binomial model for presence/absence; and Zwolinski et al. (2009) analysed acoustic data for sardine (Sardina pilchardus) based on a binomial distribution for 65 presence/absence. In each case, the primary purpose was to estimate stock abundance. However, where patchiness is mostly attributable to aggregative behaviours within suitable habitat, the density data would ideally be modelled as the outcome of a single continuous process with the observed zeroes corresponding to very low densities. Analogously, it is typically assumed that observed data are censored below some detection threshold in the analysis of rainfall or contaminant data. Values below the threshold may occur, but remain undetected. A complete spatial analysis therefore incorporates these unobserved low-level values or 'censored' data based on the observed data above the detection threshold (Stein 1992, De Oliveira 2005). The focus of inference is on the underlying distribution process that generated the data, rather than the boundary between censored and non-censored data which depends on the detection threshold and is a function of the observation process. Truncated Gaussian random fields (GRFs) are well-established in some areas of geostatistics for modelling censored data such as rainfall data (e.g. Sanso and Guenni 1999) and contaminant data (e.g. Fridley and Dixon 2007), but have been 
78 the distribution of Scottish herring (Clupea harengus) as a continuous distribution using a transformed 79 truncated GRF.

80 The method developed by Woillez et al. (2009) was founded in classical geostatistics, which does not 81 require that the dependent variable follow a known parametric distribution (Ecker 2003). In contrast, 82 likelihood-based approaches require an explicit assumption about the distribution of the dependent 83 variable as the basis for parameter estimation and analysis of uncertainty (Diggle et al. 1998). There are 84 several advantages to likelihood-based approaches for structural analysis of spatial processes and 85 parameter estimation (Ecker 2003, Roa-Ureta and Niklitschek 2007). Classical geostatistics typically 86 involves a stage-based approach to parameter estimation, whereas likelihood-based approaches allow for 87 simultaneous estimation or analysis of all parameters. In likelihood-based approaches, parameter 88 uncertainty may be evaluated through likelihood profiles, and models may be compared using information 89 criteria such as Akaike's Information Criterion (AIC) (Burnham and Anderson 2002).

90 Likelihood-based approaches support both frequentist and Bayesian approaches to inference. In 91 frequentist approaches, estimation and prediction are treated as separate processes: the geostatistical model is assumed to have fixed but unknown parameters, parameters are estimated and then point 93 estimates of the parameters are plugged back into the geostatistical model to generate predictions (Diggle 94 and Ribeiro 2007). In contrast, Bayesian prediction takes full account of uncertainty in parameters and 95 any latent variables. Uncertainty in both parameters and latent variables is characterized through posterior 96 distributions (i.e. probability distributions conditioned on the data) and prediction is based on samples 97 from the joint posterior distributions of all unknown quantities (Royle and Dorazio 2008). A Bayesian 98 approach was preferred for the analysis presented here, given uncertainty in parameters and the latent 99 distribution of interest.

100 We therefore extended the approach developed by Woillez et al. (2009) to a fully Bayesian approach. 101 Woillez et al. (2009) used non-parametric Gaussian anamorphosis (or normal-score transformation) to 102 transform the observed non-zero densities into a left-truncated GRF, while the zero densities were 103 transformed to a spike of equal values at the truncation point. It was not possible to infer the variogram of 
104 the underlying GRF directly because of this spike of equal values, so it was inferred analytically based on 105 the functional relationship between the variogram of the left-truncated GRF and the latent underlying 106 GRF (Woillez 2007). Gibbs sampling was then used to simulate Gaussian values at the locations of the 107 zero densities. In the final step, conditional geostatistical simulation was used to evaluate uncertainties 108 associated with abundance estimates derived from a combination of acoustic and trawl data.

109 In the Bayesian approach presented here, a parametric distribution was assumed for the non-zero 110 densities, and used as the basis for transformation into a left-truncated GRF. Locations with observed 111 zeroes were assumed to represent low-level values below some detection threshold (see section on 112 Observed Data below). Information on the locations of observed zeroes was incorporated into the analysis 113 using Bayesian data augmentation methods (Tanner and Wing 1987). Gibbs sampling was used to sample 114 from imputed low-level values at the locations of observed zeroes and infer the variogram. The posterior 115 densities of model parameters and imputed low-level values were then used to support prediction to a set 116 of prediction points. This Bayesian approach thus allowed for uncertainty in the variogram parameters as 117 well as imputed values at the location of observed zeroes.

118 The performance of the Bayesian integrated method was evaluated using a simulated test dataset and then 119 applied to acoustic survey data for Peruvian anchoveta (Engraulis ringens). The purpose of the testing 120 was to assess whether this method is effective at identifying the spatial parameters, density distribution, 121 and the spatial pattern of a censored process in a case where the true underlying distribution was known. 122 The purpose of the application to anchoveta was to simulate the spatial distribution of these forage fish as 123 the basis for spatial analysis of the foraging patterns of anchoveta predators (Boyd 2012). The priority 124 was therefore to reproduce the observed spatial pattern as closely as possible.

125 Peruvian anchoveta occurs in the Humboldt Current system off the coasts of Peru and northern Chile 126 (Mathisen 1989), and supports one of the largest single species fisheries in the world (Fréon et al. 2008). 127 Off Peru, anchoveta stocks are concentrated close to shore, at distances between the shoreline and 100 128 nautical miles from the coast, depending on the year (Simmonds et al. 2009). Stocks are strongly 129 associated with recently-upwelled nutrient-rich cold coastal waters (CCW) (Bertrand et al. 2004a, 
130 Swartzman et al. 2008). In most years, the CCW are spread in a broad shallow layer over the continental 131 shelf, but in some years the CCW may be restricted to isolated pockets associated with persistent 132 upwelling during warm water anomalies (Bertrand et al. 2004a). As with other small pelagic fishes, the 133 distribution of anchoveta within suitable habitat is characterized by nested aggregation structures (Fréon 134 and Misund 1999). Anchoveta exhibits strong schooling tendencies, and schools may form clusters of 135 schools within broader aggregations (Bertrand et al. 2008). Both factors, the strong association of stocks 136 with particular water masses and the formation of clusters, can lead to a high degree of patchiness in the 137 distribution of anchoveta.

\section{Methods}

\section{Observed data}

140 The Instituto del Mar del Perú (IMARPE) conducted an acoustic survey along the Peruvian coastline from 141 approximately $11.4^{\circ} \mathrm{S}$ to $12.2^{\circ} \mathrm{S}$ on the RV "Olaya" during 2-5 December 2008 and 4-7 December 2009. 142 The survey was designed to provide data on the distribution of anchoveta coincident with data on the 143 movement patterns of seabirds collected at the island group of Pescadores $\left(11.77^{\circ} \mathrm{S}\right)$. The survey 144 comprised systematic, parallel, equally-spaced, onshore-offshore transects approximately $10 \mathrm{~km}$ apart 145 (Fig. 1). The eastern end of each transect was near shore, approximately $1 \mathrm{~km}$ from the coast. The western 146 end of most transects fell within the shelf break approximately $60 \mathrm{~km}$ from the coast, with every third 147 transect continuing approximately 25 to $45 \mathrm{~km}$ beyond the shelf break to cover the observable range of 148 anchoveta. Sampling effort therefore differed between the on-shelf and off-shelf area. For the purposes of 149 this analysis, the study region was restricted to the on-shelf area covered by the survey.

150 Acoustic backscattering data were collected using a calibrated Simrad scientific echosounder (EK60) 151 operating at $120 \mathrm{kHz}$. The acoustic data were processed by IMARPE, using Echoview acoustic 152 postprocessing software (Myriax Software, Hobart, Tasmania, Australia). Acoustic backscatter was 153 identified to species based on known backscattering characteristics, ground-truthed using biological 154 samples taken from mid-water trawls during the survey (Castillo et al. 2009, Simmonds et al. 2009). The 
155 nautical area scattering coefficient (NASC) attributable to anchoveta was computed from the volume 156 backscattering strengths over 1 nautical mile transect segments (elementary distance sampling units, 157 EDSUs). Only backscatter attributed to anchoveta was used in this analysis. Geolocations were 158 transformed into the Universal Transverse Mercator coordinate system using the package rgdal() in $\mathrm{R}$ 159 (Keitt et al. 2011, R Development Core Team, 2011).

160 In both 2008 and 2009, the distributions of acoustic densities attributed to anchoveta were characterized 161 by high proportions of zero values (approximately $40 \%$ of the total in 2008 and $60 \%$ in 2009), and the 162 distributions of non-zero densities were strongly right-skewed. In December 2008, observed data indicate 163 that anchoveta was broadly distributed throughout the study region, with some areas of zero or low 164 densities in the distribution (Fig. 1a). This pattern is consistent with the hypothesis that the shelf area 165 covered by the acoustic survey mostly encompassed suitable habitat for anchoveta, and that the gaps in 166 the distribution are mostly attributable to the aggregative behaviour of anchoveta within suitable water 167 masses. In contrast, in December 2009, there were larger areas of zero or low densities (Fig. 1b). At the 168 time of 2009 survey, observed water masses in the study region (CCW and mixed CCW and subtropical 169 surface waters) provided suitable habitat for anchoveta, but the biomass of anchoveta in the study region 170 was rather low. This suggests that gaps in the distribution in 2009 were mostly attributable to a 171 combination of low regional biomass and the aggregative behaviours of anchoveta within suitable habitat.

\section{Test data}

173 Test data were generated using a process that matched the assumptions of the integrated method (see 174 below). A set of prediction points encompassing the study region was created. A single isotropic GRF 175 with exponential correlation function was simulated to the 2008 transect locations and the prediction 176 points (Fig. 2a). The parameters governing spatial autocorrelation were chosen to be close to values 177 indicated in exploratory analysis of the observed anchoveta data for 2008. Due to the random nature of 178 the process, the realised parameters of a simulated GRF are not expected to correspond exactly to the 179 simulation parameters. The mean and variance of the test data were standardized for convenience. Two 
censored datasets were then derived from the single test dataset by censoring values less than a censoring point, defined as (i) the $40^{\text {th }}$ percentile (Fig. 2b) and (ii) the $60^{\text {th }}$ percentile, corresponding to the proportion of observed zeroes in the anchoveta data for 2008 and 2009 respectively. Values at the transect locations were then extracted to form two estimation datasets (Fig. 2c), leaving values at the prediction points to provide two evaluation datasets.

\section{Geostatistical model}

A standard geostatistical model (following Diggle and Ribeiro 2007) may comprise a trend surface, a spatial signal process, and additional non-spatial or residual variance:

(1) $\quad Y_{i} \sim \sum_{j=1}^{J} \beta_{j} D_{j i}+S_{i}+\varepsilon_{i}$

where $Y$ is a random variable observed at a set of sampling locations $i=1 \ldots . \mathrm{N} ; D_{\mathrm{ji}}$ are $\mathrm{j}=1 \ldots \mathrm{J}$

measured spatial coordinates or environmental covariates and $\beta_{\mathrm{j}}$ are the corresponding parameters; $S_{\mathrm{i}}$ is the spatial signal process, $E\left[S_{i}\right]=0$; and $\varepsilon_{\mathrm{i}}$ are independent and identically distributed with zero mean and non-spatial variance $\tau^{2}$. The theoretical variogram is used to characterize the spatial signal process. In the stationary and isotropic case, this simplifies to:

$$
V(h)=\sigma^{2}[1-\rho(h)]
$$

where $\sigma^{2}$ is the variance of the spatial signal process (the 'partial sill'), which is constant in the stationary case; $\rho(h)$ is the correlation function; and $h$ is the absolute Euclidean distance between two locations. In the case of second-order stationarity, the covariance depends only on the absolute distance between two locations. The correlation function, $\rho(h)$, is usually chosen from a range of standard families which are known to be positive definite, but are otherwise sufficiently flexible to meet the needs of geostatistical data (Diggle and Ribeiro 2007). Based on exploratory analysis of the acoustic survey data, an exponential correlation function was assumed throughout the analysis presented here:

$$
\rho(h)=\exp \left(-\frac{h}{\varphi}\right)
$$

where the scale parameter, $\varphi>0$, is approximately one third of the practical range, i.e. the distance at which correlation is 0.05 . Spatial autocorrelation may be isotropic or anisotropic. However, in this case, 
205 the spatial distribution was assumed to be isotropic (i.e. spatial continuity is similar in all directions) as 206 the study region was small. We further assumed a constant trend or mean (i.e. $E\left(Y_{i}\right)=\beta_{j} D_{j i}=\mu$ ). The 207 restricted maximum likelihood estimator for a stationary GRF is:

208

$$
L L\left(\varphi, \sigma^{2}, \tau^{2}\right)=-0.5\left\{n \cdot \log (2 \pi)+\log \left|\sigma^{2} V_{\varphi}\right|+\log \left|D^{\prime}\left(\sigma^{2} V_{\varphi}\right)^{-1} D\right|+Z^{\prime}\left(\sigma^{2} V_{\varphi}\right)^{-1} Z\right\}
$$

where $n$ is the number of observations; $V_{\varphi}=R_{\varphi}+v^{2} I, R_{\varphi}$ is the correlation matrix; $v^{2}=\frac{\tau^{2}}{\sigma^{2}} ; D$ is a vector of $1 \mathrm{~s}$ in the case of a constant trend; and $Z$ is the vector of observed minus expected values (see Diggle and Ribeiro, 2007, p. 117).

\section{Bayesian data augmentation, inference, and posterior prediction}

\section{Test data}

214 For the two estimation datasets, the variable underlying the censored and non-censored values was known 215 to follow an isotropic GRF with zero mean (i.e. $\mu=0$ ), and the sampling distribution on which the log216 likelihood in Equation 4 was based. Bayesian data augmentation (Tanner and Wing 1987) was used to 217 impute values at the locations of the censored data. Bayesian data augmentation is useful when the data 218 comprise a combination of observed and unobserved or latent data, and the posterior density of the 219 parameters is difficult to calculate without the latent data but would be comparatively straight-forward if 220 the latent data were fully observed. The Bayesian data augmentation solution is to generate many sets of 221 values for the latent data, and then approximate the posterior density over the many sets of imputed values 222 (see De Oliveira 2005 for a relevant geological example). Here, a Gibbs sampler was used to impute the 223 censored values and sample from the posterior densities of the parameters $\varphi, \sigma^{2}, \tau^{2}$. Imputed values were 224 drawn from a continuous uniform distribution with the upper bound set at the censoring point 225 corresponding to the dataset being analysed and the lower bound set to a value (-5) with probability 226 density $<0.00001$ under the standard Gaussian distribution. Following Diggle and Ribeiro (2007), both $\varphi$ 227 and $v^{2}$ were discretized to reduce computation time. The priors for parameters $\varphi, \sigma^{2}, v^{2}$ were assumed to be independent. For $\varphi>0$, a uniform discrete prior $\{1,2,3, \ldots 50\}$ was chosen, with the upper limit set to 
$22950 \mathrm{~km}$. For $v^{2}=\frac{\tau^{2}}{\sigma^{2}}$, a uniform discrete prior $\{0,0.1,0.2, \ldots 1\}$ was used. The prior for the inverse of $\sigma^{2}$ 230 was $\Gamma(0.1,0.1)$. The Gibbs sampler was implemented in JAGS (Just Another Gibbs Sampler, Plummer 231 2003). For each estimation dataset, two parallel chains were generated with a thinning rate of 50. After 232 discarding the first 250 parameter sets, 250 parameter sets were saved from each chain. Convergence was 233 evaluated using the Geweke and Gelman-Rubin diagnostic statistics (Geweke 1992, Gelman and Rubin 234 1992, Brooks and Gelman 1998).

235 Posterior predictions were then generated to the set of prediction points following development of the 236 posteriors for $\varphi, \sigma^{2}, v^{2}$. One hundred parameter sets (including imputed values) were sampled at random 237 from the two chains for each estimation dataset. For each sampled parameter set, conditional geostatistical 238 Gaussian simulation (Goovaerts 1997) was used to generate posterior densities of values at the prediction 239 points, based on a single realisation of the GRF for each of the 100 parameter sets. The posterior 240 predictions were conditioned on the non-zero densities and the locations of the censored data, while the 241 model parameters and the imputed values at the locations of censored data were treated as uncertain. For 242 the posterior predictions, values less than the $40^{\text {th }}$ or $60^{\text {th }}$ percentile of the standard Gaussian distribution 243 were then censored as appropriate to check whether the proportion of censored values in the posterior 244 matched the true population value in the test data. ( $\mathrm{R}$ code for reproducing the simulations and Bayesian 245 data augmentation, inference and prediction is available in the Supplementary Material.)

\section{Observed data}

247 For the observed data, the first step in the modelling process was to transform the non-zero densities for 248 each survey year into a left-truncated Gaussian distribution with the censoring point corresponding to the 249 observed proportion of zero values. In likelihood-based geostatistics, zero-truncated right-skewed 250 continuous distributions may be modelled using a linear model following Box-Cox transformation (Box 251 and Cox 1964). Preliminary analysis indicated that log-transformation provided the best fit to the non252 zero acoustic densities (Fig. 3). Following log-transformation, the Anderson-Darling test for normality 253 indicated that the observed data were consistent with a Gaussian distribution ( $\mathrm{p}$-value $=0.815$ ) in 2009 
254 but not in 2008 (p-value $=0.019$ ). Nonetheless, a log-Gaussian distribution was assumed in both cases, 255 and the effects of this assumption are discussed further below. For each survey year, probability points 256 were calculated for the non-zero densities based on a log-Gaussian distribution and then rescaled to allow 257 for the observed proportion of zero values in each year, i.e. the rescaled probability points fell within the 258 range $\sim 0.4-1$ for the 2008 data and $\sim 0.6-1$ for the 2009 data. The non-zero densities were then 259 transformed into standard Gaussian space with zero mean and unit variance using the rescaled probability 260 points. ( $\mathrm{R}$ code for transforming a variable to a truncated Gaussian distribution is available in the 261 Supplementary Material.) The transformed variable (combining transformed non-zero densities and latent 262 values corresponding to the observed zeroes) was then assumed to follow an isotropic GRF with zero 263 mean (i.e. $\underline{\mu}=0$ ) and log-likelihood as in Equation 4. Environmental covariates were not included in the 264 analysis presented here, but could have been included as per Equation 1 (see also Diggle and Ribeiro, 2652007 , p. 117).

266 Bayesian data augmentation, inference, and posterior prediction were implemented as for the test data, 267 with the following adjustments. The upper limit for $\varphi>0$ was extended to $100 \mathrm{~km}$, approximately the 268 maximum distance between survey locations. The step size in the uniform discrete prior for $v^{2}=\frac{\tau^{2}}{\sigma^{2}}$ was 269 refined to $\{0,0.025,0.05, \ldots 1\}$. For each observed dataset, two parallel chains were generated with a 270 thinning rate of 200. After discarding the first 250 parameter sets, 250 parameter sets were saved from 271 each chain. The 2009 survey did not extend as far south as the 2008 survey (Fig. 1), so the set of 272 prediction points for 2009 was cropped to avoid extrapolation from the observed data. Finally, values less 273 than the predefined censoring point were censored, and the vector of non-censored data was back274 transformed to the observed data space using the inverse of the procedure described above.

\section{Results}

276 Performance of the Bayesian integrated method was assessed in terms of the posterior densities for the 277 spatial parameters, and the density distribution, spatial pattern, and overall spatial distribution of posterior 278 predictions. 


\section{Test data}

\section{Posterior densities of the spatial parameters}

281 Samples from the posterior densities of the spatial parameters of the uncensored and censored test data are 282 shown in Figure 4. The posterior density distributions are similar, with some increase in uncertainty as $28340 \%$ and $60 \%$ of values are censored.

\section{Density distributions of the posterior predictions}

285 In the case of the test data, the mean and variance of the simulated GRF are known (Fig. 5). Differences 286 between descriptive statistics for the estimation and evaluation datasets are indicative of sampling error 287 and are not attributable to the analytical method. In the case of the variance, the difference is partly 288 structural. Spatial autocorrelation implies that the variance between points that are close together is less 289 than the variance between points that are far apart. The prediction locations are interpolated among the 290 transect locations - each prediction location therefore has more close neighbours and the overall variance 291 is consequently lower.

292 The mean and variance of the simulated GRF are well reproduced in posterior predictions, as is the 293 proportion of values that fall below the censoring point and the density distribution of non-censored 294 values when $40 \%$ of values are censored (Fig. 5a). The mean and variance of the simulated GRF are less 295 well reproduced when $60 \%$ of values are censored (Fig. 5b). Nevertheless, the proportion of values that 296 fall below the censoring point and the density distributions of the non-censored values are well297 reproduced (Fig. 5b).

\section{Spatial pattern of the censoring process and of the non-censored values}

299 The performance of the Bayesian integrated method in terms of reproducing the spatial pattern of the 300 underlying process can be evaluated by comparing boxplots of empirical variograms derived from the 301 posterior predictions with the empirical variogram of the simulated GRF after censoring (Fig. 6). 
302 Overall, the spatial patterns in the posterior predictions correspond well to the patterns in the evaluation 303 datasets, especially at short distances. Spatial autocorrelation in the censoring process (approximating 304 presence/absence) is well-reproduced, (Fig. 6a). Sampling error is more pronounced for the non-censored 305 values (approximating conditional abundance). The variograms calculated from posterior predictions of 306 non-censored values generally fall between variograms calculated from values at the prediction points and 307 transect locations.

\section{Overall spatial distribution}

309 Figure 7 shows the simulated GRF after censoring, together with sample posterior predictions. The 310 posterior predictions reproduce the spatial distribution of the censored GRF, in terms of the distribution of 311 censored values and peaks in non-censored values.

\section{Observed data}

\section{Posterior densities of the spatial parameters}

314 Samples from the posterior densities of the spatial parameters based on analysis of the acoustic survey 315 data for anchoveta are shown in Figure 8. Overall, the posterior distributions for the spatial parameters 316 compare well to those for the test data after allowing for the broader prior distribution for the scaling 317 parameter, $\phi$, used in the analysis of observed data. The posterior distribution for the non-spatial 318 variance, $\tau^{2}$, is notably more concentrated.

319 The posterior densities indicate several differences in the spatial process underlying the distribution of 320 anchoveta in 2008 versus 2009 (Fig. 8). Posterior densities of the scaling parameter, $\varphi$ indicate that the 321 distance over which spatial autocorrelation decays was likely longer in 2009 than 2008, consistent with 322 fewer larger clusters in 2009. Posterior densities of the variance parameters indicate that non-spatial 323 variance was relatively low and hence the spatial signal represented a greater proportion of the overall 324 variance in 2009, consistent with better-defined clusters. 


\section{Density distributions of the posterior predictions}

326 In posterior predictions based on the 2008 data (Fig. 9a), the proportion of zero densities and the mean of 327 the non-zero densities correspond closely to the data observed at transect locations. The mode of the 328 variance of non-zero densities also corresponds to the variance at transect locations, but the variance 329 ranges quite broadly among posterior predictions. In posterior predictions based on the 2009 data (Fig. $3309 \mathrm{~b}$ ), the proportion of zero densities tends to be higher than observed at transect locations, while the mean 331 and variance of non-zero densities correspond more closely to data observed at transect locations. 332 Posterior predictions effectively reproduce the density distribution of non-zero values observed in 2009 333 (Fig 9b). In contrast, posterior predictions based on the 2008 data do not reproduce the spike of low 334 values in the observed data because this is not consistent with the assumed log-Gaussian distribution (Fig $3359 a)$.

\section{Spatial pattern of presence/absence and conditional abundance}

337 Comparison of empirical indicator variograms derived from the posterior predictions with empirical 338 indicator variograms for the transect data (Fig. 10a) indicates that the non-spatial variance in the 339 presence/absence process is over-estimated, especially in 2008. The contrast with the test data is striking, 340 and suggests that the edges of anchoveta clusters are more consolidated than in the test data.

\section{Overall spatial distribution}

342 Figure 11 shows the observed transect data for 2008 and 2009, together with sample posterior predictions 343 for each year. The posterior predictions reproduce the overall spatial distribution of the observed data in 344 terms of the distribution of censored values and peaks in non-censored values. Anchoveta was broadly 345 distributed across the study region with some gaps in the distribution in the northwest and the southeast in 346 2008, whereas it was mostly concentrated nearshore, with some more diffuse clusters near the shelf break 347 in 2009. 


\section{Discussion}

\section{A new modelling framework}

350 The appropriate method for spatial analysis of the distribution of small pelagic fish depends on the

351 processes underlying the observed distribution and the purpose of the analysis. Two-stage models are

352 appropriate where the processes leading to gaps in the distribution are distinct from those determining 353 conditional abundance. Here, we developed an approach suitable for modelling distributions in which 354 patchiness is largely attributable to the aggregation of small pelagic fish within a region of suitable 355 habitat. Following Woillez et al. (2009), the patchy distribution was modelled as the outcome of a 356 continuous but censored aggregation process, based on a transformed truncated GRF. We developed a 357 fully Bayesian approach, using Bayesian data augmentation to impute censored values and accounting for 358 uncertainty in the parameters of the spatial distribution.

359 Performance testing revealed that this Bayesian integrated method was effective at identifying the spatial 360 parameters of a simulated GRF from censored data at transect locations, and at reproducing the density 361 distribution of non-censored data. Performance deteriorated slightly when $60 \%$ of values were censored. 362 The spatial pattern of the censoring process and the non-censored values was well-reproduced in posterior 363 predictions, together with the spatial and density distribution of the censored variable. While the posterior 364 predictions are based on samples from a wide range of parameter values, the spatial distributions that 365 emerge are consistent with the simulated GRF across posterior predictions. This is attributable to 366 conditioning on the values at transect locations, which provide a consistent skeleton on which posterior 367 predictions are constructed.

368 In performance testing, the data-generating process was known to be a censored isotropic GRF, whereas 369 the spatial processes underlying the observed data were unknown. Differences in performance of the 370 Bayesian integrated method when applied to test data versus observed data point towards possible 371 deviations from the assumptions underlying implementation of the method. Poor identification of spatial 372 parameters, for example, would suggest that the spatial process underlying the observed data was poorly 
373 represented by a censored GRF. Overall, the Bayesian integrated method performed well when applied to 374 the anchoveta data for both 2008 and 2009, with two notable exceptions.

375 First, the Bayesian integrated method effectively reproduced the spatial pattern of the observed non-zero 376 densities, but the non-spatial variance in the censoring process was notably higher in posterior predictions 377 than in the transect data, especially for 2008. This suggests that the edges of clusters are more clearly378 defined than shown in the posterior predictions, while the interior spatial structure is better-represented. 379 One hypothesis to explain the relatively low contribution of non-spatial variance to the presence/absence 380 process would be differences in the inherent aggregative behaviours underpinning presence/absence 381 versus conditional abundance. The impulse that underlies the formation of schools by small pelagic fish 382 may be strong, contributing to low non-spatial variance, whereas spatial structure within schools may be 383 more heterogeneous contributing to higher non-spatial variance (Gerlotto and Paramo 2003, Bertrand et 384 al. 2008).

385 Second, the observed non-zero densities were not consistent with a log-Gaussian distribution in 2008. 386 Consequently, the density distributions of posterior predictions more closely follow a Gaussian 387 distribution than the observed log-transformed data for 2008. In likelihood-based approaches, effective 388 inference depends on how well the assumed distribution approximates the true distribution of the variable 389 of interest (Isaaks and Srivastava 1989, Matheron 1978). Where the observed data do not follow a 390 parametric distribution, researchers must choose between a parametric and non-parametric approach. This 391 choice will depend on the purpose of analysis, the extent of deviations from a parametric distribution, and 392 an assessment of whether these are representative of the true distribution or reflect sampling error. 393 Analysis of the observed anchoveta data using the Bayesian integrated method following non-parametric 394 transformation is presented in the Supplementary Material. Comparison of the results following 395 parametric and non-parametric transformations indicates that the posterior densities for the spatial 396 parameters and spatial pattern are very similar. However, when the analysis was extended to include 397 simple estimates of regional abundance following back-transformation, substantial differences emerged 398 attributable to the deviations of the observed sample distribution from a true log-Gaussian distribution. 
399 The primary purpose of our analysis was to simulate the spatial distribution of small pelagic fish as the 400 basis for analysing the foraging patterns of their predators, and we chose to use a fully parametric 401 approach. Other researchers may choose a non-parametric transformation, especially for the purposes of 402 abundance estimation.

\section{Comparison with existing approaches}

404 Where the primary focus of spatial analysis is the presence/absence process rather than conditional 405 abundance, alternative geostatistical methods may be considered. Indicator simulation (Goovaerts 1997) 406 provides one option for simulating presence/absence (e.g. Walline 2007), but falls outside the likelihood407 based framework so the advantages of a likelihood-based approach for structural analysis would be lost. 408 In likelihood-based geostatistics, the conventional approach to modelling a presence/absence process is 409 based on a binomial model (e.g. Roa-Ureta and Niklitschek 2007). However, a major challenge for spatial 410 analysis based on a single Bernoulli trial at each location, as in the anchoveta data, is the limited 411 information available to estimate the spatial signal (Diggle and Ribeiro 2007). Repeated surveys may be 412 necessary to generate sufficient information to estimate the parameters of the binomial model. This 413 strategy was shown to be useful in analysis of Patagonian squid (Loligo gahi) (Roa-Ureta and Niklitschek 414 2007), but its effectiveness will depend on the time interval between surveys versus the mobility of the 415 stock. The binomial geostatistical model is based on the assumption that the spatial signal process takes 416 the form of a hypothetical GRF which is transformed into a probability field through the inverse logit 417 function. The response variable is then assumed to follow a Bernoulli distribution based on the 418 probability field. In the final step, an indicator value is drawn independently for each location based on 419 the inferred spatially-dependent probability field. This step is inappropriate for species with strong 420 inherent aggregative behaviours, such as anchoveta. For these species, presence/absence at a location is 421 not independent of presence/absence at neighbouring locations, even after the underlying probability of 422 occurrence has been taken into account. Binomial simulation leads to a more fragmented pattern than the 
423 integrated method (Boyd 2012), and is inappropriate for species with inherent aggregative behaviours that 424 extend beyond the scale of analysis.

425 In conclusion, truncated GRFs are well-established in other fields of geostatistics, but have only recently 426 been applied to fisheries data. The analysis presented here demonstrated that Bayesian posterior 427 prediction using a truncated GRF can be effective at reproducing the patchy spatial distribution of 428 anchoveta. This method is broadly applicable to the patchy distributions of small pelagic fish and krill 429 within suitable habitat and the three main purposes of geostatistical analysis for fisheries ecology 430 identified in the Introduction. There are several differences in the overall spatial distribution simulated 431 using the Bayesian integrated method compared to a two-stage model. In two-stage models, no 432 information is passed from the presence/absence stage to the conditional abundance stage on the 433 probability of occurrence at a location. All points with predicted presence are treated as equal in the 434 second stage, so high values may be predicted in areas with low probability of occurrence. In contrast, all 435 information is retained throughout the posterior prediction process in the integrated method. As a result, 436 the integrated method generally predicted low values, if any, in areas near where the acoustic survey 437 returned zero values. The integrated method may prove more efficient for structural analysis and 438 inference on the relationship between the spatial distributions of fish and environmental covariates 439 because the analysis is not split into two separate and independent stages. Furthermore, in the integrated 440 method, predicted values near the edge of clusters are constrained by low neighbouring densities whereas 441 they are unconstrained by nearby zero values in two-stage models. The greater spatial structure in the 442 integrated method is therefore expected to reduce the variance in posterior predictions of stock 443 abundance. The purpose of our analysis was to simulate the spatial structure of aggregations of anchoveta, 444 as the basis for spatial analysis of the foraging patterns of anchoveta predators (Boyd 2012). As tracking 445 data on marine predators and fishing fleets become more widely available, we anticipate that similar 446 analyses of the spatial pattern of forage fish, their natural predators, and fishing fleets will become an 447 important tool for strengthening understanding of spatial structure and function in marine ecosystems as 448 an input to ecosystem-based management. 


\section{Acknowledgements}

450 The authors would like to thank J. Horne and the Fisheries Acoustics Research laboratory at the

451 University of Washington (USA) for conceptual discussion and feedback. We would also like to thank

452 three anonymous reviewers for their valuable comments which led to substantial improvements to

453 the analysis and manuscript. Data was collected by the Instituto del Mar del Perú (IMARPE, Peru). This

454 work is a contribution to the cooperative agreement between the Instituto del Mar del Perú (IMARPE),

455 the Institut de Recherche pour le Développement (IRD), the Agence Nationale de la Recherche (ANR)

456 project 'Top Predators as Indicators of Exploited Marine Ecosystem dynamics' (TOPINEME), and of the 457 International Joint Laboratory DISCOH. 


\section{References}

Bertrand, A., Gerlotto, F., Bertrand, S., Gutiérrez, M., Alza, L., Chipollini, A., Diaz, E., Espinoza, P., Ledesma, J., Quesquen, R., Peraltilla, S., and Chavez, F. 2008. Schooling behaviour and environmental forcing in relation to anchoveta distribution: An analysis across multiple spatial scales. Progress in Oceanography 79(2-4): 264-277.

Bertrand, A., Segura, M., Gutiérrez, M., and Vasquez, L. 2004a. From small-scale habitat loopholes to decadal cycles: a habitat-based hypothesis explaining fluctuation in pelagic fish populations off Peru. Fish and Fisheries 5(4): 296-316.

Bertrand, S., Diaz, E., and Niquen, M. 2004b. Interactions between fish and fisher's spatial distribution and behaviour: an empirical study of the anchovy (Engraulis ringens) fishery of Peru. ICES Journal of Marine Science 61(7): 1127-1136.

Box, G.E.P., and Cox, D.R. 1964. An analysis of transformations. Journal of the Royal Statistical Society, Series B 26(2): 211-252.

Boyd, C. 2012. The Predator's Dilemma: Investigating the responses of seabirds to changes in the abundance and distribution of small pelagic prey, School of Aquatic and Fishery Sciences, University of Washington, Seattle, WA.

Brooks, S.P., and Gelman, A. 1998. General methods for monitoring convergence of iterative simulations. Journal of Computational and Graphical Statistics 7(4): 434-455.

Burnham, K.P., and Anderson, D.R. 2002. Model Selection and Multimodel Inference: A Practical Information-Theoretic Approach. Springer-Verlag, New York.

Castillo, R., Peraltilla, S., Aliaga, A., Flores, M., Ballón, M., Calderón, J., and Gutiérrez, M. 2009.

Protocolo técnico para la evaluación acústica de las áreas de distribución y abundancia de recursos pelágicos en el mar peruano. Informe Instituto del Mar Perú 36(1-2): 7-28.

Ciannelli, L., Fauchald, P., Chan, K.S., Agostini, V.N., and Dingsor, G.E. 2008. Spatial fisheries ecology: Recent progress and future prospects. Journal of Marine Systems 71(3-4): 223-236. 
484 De Oliveira, V. 2005. Bayesian inference and prediction of Gaussian random fields based on censored 485 data. Journal of Computational and Graphical Statistics 14(1): 95-115.

486 Diggle, P.J., and Ribeiro, P.J. 2007. Model-based Geostatistics. Springer, New York.

487 Diggle, P.J., Tawn, J.A., and Moyeed, R.A. 1998. Model-based geostatistics. Journal of the Royal 488 Statistical Society Series C-Applied Statistics 47: 299-326.

489 Ecker, M.D. 2003. Geostatistics: past, present and future. In Encyclopedia of Life Support Systems 490 (EOLSS). Developed under the Auspices of the UNESCO. [http://www.eolss.net] EOLSS Publishers, 491 Oxford, UK.

492 Fréon, P., Bouchon, M., Mullon, C., Garcia, C., and Niquen, M. 2008. Interdecadal variability of 493 anchoveta abundance and overcapacity of the fishery in Peru. Progress in Oceanography 79(2-4): 401494412.

495 Fréon, P., Cury, P., Shannon, L., and Roy, C. 2005. Sustainable exploitation of small pelagic fish stocks 496 challenged by environmental and ecosystem changes: A review. Bulletin of Marine Science 76(2): 385497462.

498 Fréon, P., and Misund, O.A. 1999. Dynamics of pelagic fish distribution and behavior: effects on fisheries 499 and stock assessment. Blackwell Science, Oxford, UK.

500 Fridley, B.L., and Dixon, P. 2007. Data augmentation for a Bayesian spatial model involving censored 501 observations. Environmetrics 18(2): 107-123.

502 Gelman, A., and Rubin, D.B. 1992. Inference from iterative simulation using multiple sequences. 503 Statistical Science 7: 457-511.

504 Gerlotto, F., and Paramo, J. 2003. The three-dimensional morphology and internal structure of clupeid 505 schools as observed using vertical scanning multibeam sonar. Aquatic Living Resources 16: 113-122.

506 Geweke, J. 1992. Evaluating the accuracy of sampling-based approaches to calculating posterior 507 moments. In Bayesian Statistics 4. Edited by J.M. Bernado, J.O. Berger, A.P. Dawid and A.F.M. Smith. 508 Oxford University Press, Oxford, UK. pp. 169-119 
509 Gimona, A., and Fernandes, P.G. 2003. A conditional simulation of acoustic survey data: advantages and 510 potential pitfalls. Aquatic Living Resources 16: 123-129.

511 Goovaerts, P. 1997. Geostatistics for Natural Resources Evaluation. Oxford University Press, New York. 512 Isaaks, E.H., and Srivastava, R.M. 1989. An Introduction to Applied Geostatistics Oxford University 513 Press, New York.

514 Keitt, T.H., Bivand, R., Pebesma, E., and Rowlingson, B. 2011. rgdal: Bindings for the Geospatial Data 515 Abstraction.

516 Lezama-Ochoa, A., Ballon, M., Woillez, M., Grados, D., Irigoien, X., and Bertrand, A. 2011. Spatial 517 patterns and scale-dependent relationships between macrozooplankton and fish in the Bay of Biscay: an 518 acoustic study. Marine Ecology-Progress Series 439: 151-168.

519 Matheron, G. 1978. Estimer et Choisir: essai sur la pratique des probabilités, Center de Géostatistique, 520 Ecole Nationale Supérieure des Mines de Paris, Paris.

521 Mathisen, O.A. 1989. Adaptation of the anchoveta (Engraulis ringens) to the Peruvian upwelling system. 522 In The Peruvian upwelling ecosystem: dynamics and interactions. Edited by D. Pauly, P. Muck, J. Mendo 523 and I. Tsukayama. pp. 220-234.

524 Maunder, M.N., and Punt, A.E. 2004. Standardizing catch and effort data: a review of recent approaches. $525 \quad$ Fisheries Research 70(2-3): 141-159.

526 Petitgas, P. 1993. Geostatistics for fish stock assessments - a review and an acoustic application. ICES 527 Journal of Marine Science 50(3): 285-298.

528 Plummer, M. 2003. JAGS: A Program for Analysis of Bayesian Graphical Models Using Gibbs 529 Sampling. In Proceedings of the 3rd International Workshop on Distributed Statistical Computing, 530 Vienna, Austria.

531 R Development Core Team. 2011. R: A language and environment for statistical computing. R 532 Foundation for Statistical Computing, Vienna, Austria.

533 Rivoirard, J., Simmonds, J., Foote, K.G., Fernandes, P., and Bez, N. 2000. Geostatistics for estimating 534 fish abundance. Blackwell Science, Oxford, UK. 
535 Roa-Ureta, R., and Niklitschek, E. 2007. Biomass estimation from surveys with likelihood-based 536 geostatistics. ICES Journal of Marine Science 64(9): 1723-1734.

537 Royle, J.A., and Dorazio, R.M. 2008. Hierarchical Modeling and Inference in Ecology. Academic Press, 538 Amsterdam.

539 Sanso, B., and Guenni, L. 1999. Venezuelan rainfall data analysed by using a Bayesian space-time model. 540 Journal of the Royal Statistical Society Series C-Applied Statistics 48: 345-362.

541 Simmonds, E.J., Gutiérrez, M., Chipollini, A., Gerlotto, F., Woillez, M., and Bertrand, A. 2009.

542 Optimizing the design of acoustic surveys of Peruvian anchoveta. ICES Journal of Marine Science 66(6): $543 \quad 1341-1348$.

544 Simmonds, E.J., and MacLennan, D.N. 2005. Fisheries Acoustics. Blackwell Science, Oxford, UK. 545 Stein, M.L. 1992. Prediction and Inference for Truncated Spatial Data. Journal of Computational and 546 Graphical Statistics 1: 91-110.

547 Swartzman, G., Bertrand, A., Gutiérrez, M., Bertrand, S., and Vasquez, L. 2008. The relationship of 548 anchovy and sardine to water masses in the Peruvian Humboldt Current System from 1983 to 2005. 549 Progress in Oceanography 79(2-4): 228-237.

550 Tanner, M.A., and Wing, H.W. 1987. The calculation of posterior distributions by data augmentation. 551 Journal of the American Statistical Association 82(398): 528-540.

552 Walline, P.D. 2007. Geostatistical simulations of eastern Bering Sea walleye pollock spatial distributions, 553 to estimate sampling precision. ICES Journal of Marine Science 64(3): 559-569.

554 Woillez, M. 2007. Contributions géostatistiques à la biologie halieutique, Ecole Nationale Supérieure des 555 Mines de Paris. France.

556 Woillez, M., Rivoirard, J., and Fernandes, P.G. 2009. Evaluating the uncertainty of abundance estimates 557 from acoustic surveys using geostatistical simulations. ICES Journal of Marine Science 66(6): 1377-1383. 558 Zwolinski, J., Fernandes, P.G., Marques, V., and Stratoudakis, Y. 2009. Estimating fish abundance from 559 acoustic surveys: calculating variance due to acoustic backscatter and length distribution error. Canadian 560 Journal of Fisheries and Aquatic Sciences 66(12): 2081-2095. 


\section{$561 \quad$ Figure legends}

562 Figure 1. Proportional representation of acoustic backscatter $\left(\mathrm{m}^{2}\right.$ per nautical mile ${ }^{2}$ ) in (a) 2008 and (b) 563 2009. Elementary distance sampling units (EDSUs) are marked by crosses (zero values for anchoveta) 564 and circles (positive values, diameter of the circles proportional to the logarithm of relative anchoveta 565 abundance). The shelf break (200 $\mathrm{m}$ isobaths) is indicated by the dashed line.

566 Figure 2. (a) A standard isotropic GRF was simulated to the 2008 transect locations and prediction 567 points. Darker shades indicate higher densities. (b) Values less than the $40^{\text {th }}$ percentile were censored. 568 White indicates censored values. (c) The transect locations were extracted. Steps (b) and (c), were 569 repeated with values less than the 60th percentile censored (not shown).

570 Figure 3. Histograms of the observed log-transformed non-zero acoustic densities by EDSU for (a) 2008 571 and (b) 2009, with Gaussian density distributions overlaid.

572 Figure 4. Samples from the posterior densities for the test data when (a) $0 \%$, (b) 40\%, and (c) 60\% of 573 values are censored. Parameter values used to generate the test data are indicated by dashed vertical lines. 574 Figure 5. Posterior densities for the test data when (a) $\sim 40 \%$, and (b) $\sim 60 \%$ of values are censored: from 575 left to right, the mean of the simulated GRF prior to censoring; the variance of the simulated GRF prior to 576 censoring; and the proportion of values censored. In each case, the dashed vertical line represents the 577 population value estimated from the evaluation points, and the dotted vertical line represents the sample 578 value from the estimation datasets. In the far right panel, histograms show the density distributions of the 579 simulated GRF after censoring. The solid line shows the mean; dashed lines show the 2.5th and 97.5th 580 percentile of the corresponding posterior densities.

581 Figure 6. Quantitative summaries of the spatial pattern of (a) the censoring process (approximating 582 presence/absence) and (b) the non-censored values (approximating conditional abundance). Box plots 583 represent empirical variograms computed from posterior predictions. Dashed lines represent empirical 584 variograms for the simulated GRF; dotted lines represent empirical variograms for the transect data. 
585 Figure 7. The simulated GRF after censoring when (a) $\sim 40 \%$ and (b) $\sim 60 \%$ of values are censored (far 586 left panel), together with three sets of sample posterior predictions. Darker shades indicate higher 587 densities in quantiles and white indicates censored values.

588 Figure 8. Samples from the posterior densities for observed data on anchoveta from (a) 2008 and (b) 589 2009. From left to right, panels show the scaling parameter, spatial variance, and non-spatial variance.

590 Figure 9. Samples from the posterior densities for observed data on anchoveta from (a) 2008 and (b) 591 2009. From left to right, panels show the proportion of values censored, the mean of non-zero densities, 592 and the variance of non-zero densities, with the dotted vertical line representing the value from the 593 transect data. In the far right panel, histograms show the distributions of the observed log-transformed 594 non-zero densities; the solid line shows the mean; dashed lines show the 2.5 th and 97.5 th percentile of the 595 corresponding posterior densities.

596 Figure 10. Quantitative summaries of the spatial pattern of observed data on anchoveta of (a) 597 presence/absence and (b) conditional abundance. Box plots represent empirical variograms computed 598 from posterior predictions. Dotted lines represent empirical variograms for the observed data.

599 Figure 11. The observed transect data from (a) 2008 and (b) 2009, together with three sets each of sample 600 posterior predictions. Darker shades indicate higher densities in quantiles and white indicates censored 601 values. 


\section{Page 27 of 37}
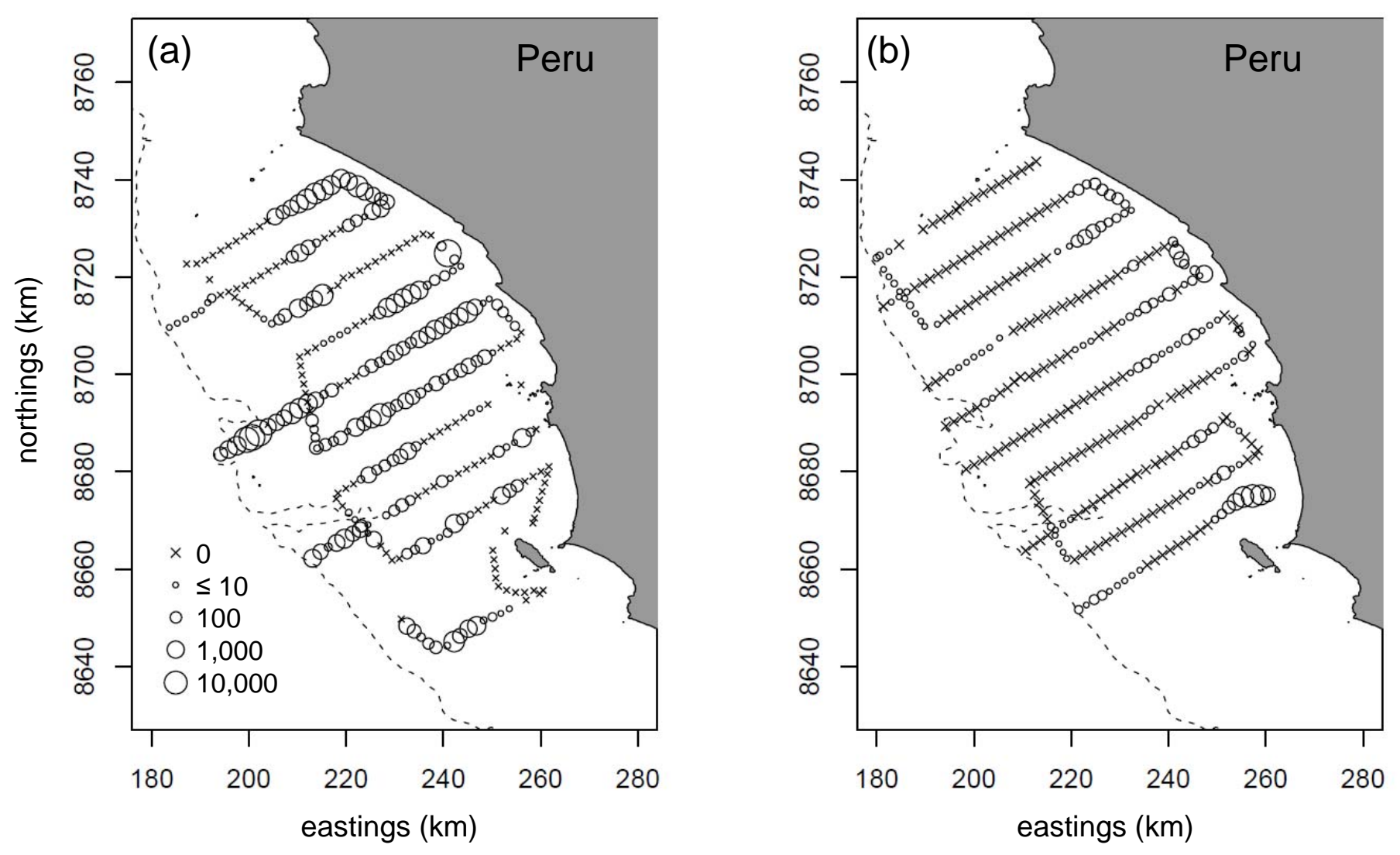
(a)

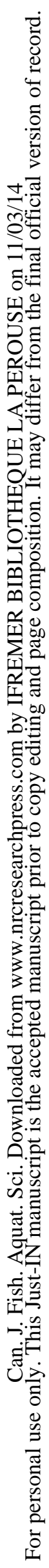

(b)

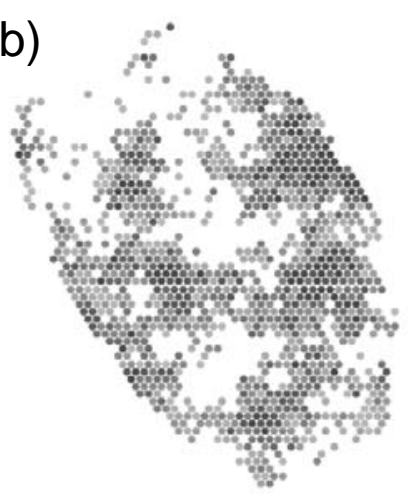

(c)

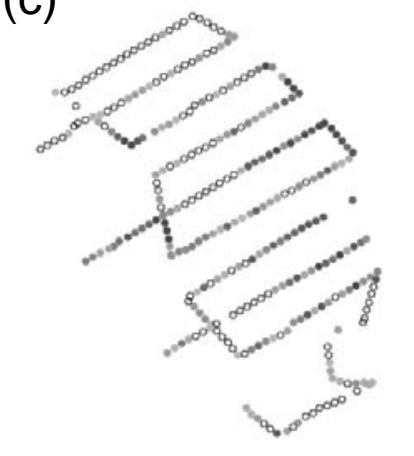




\section{Page 29 of 37}
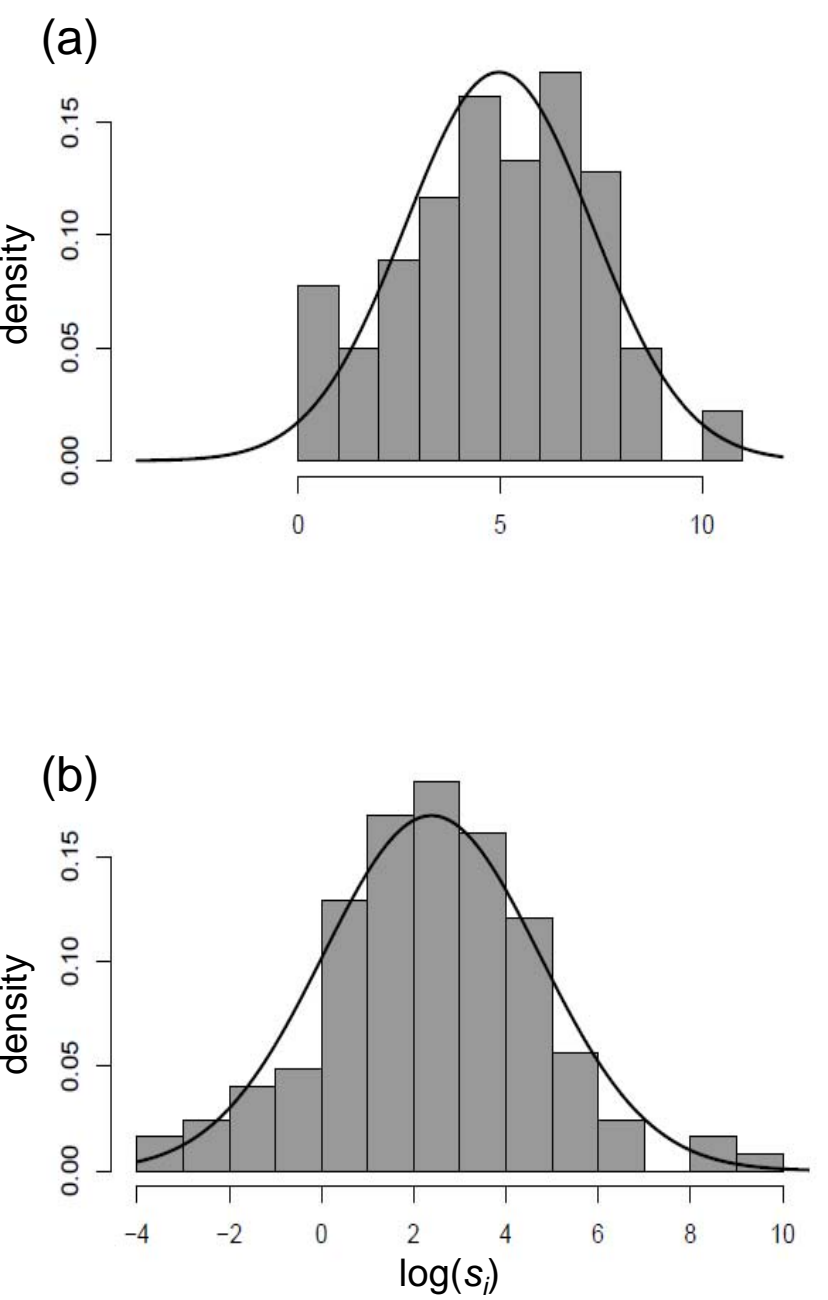
(a)
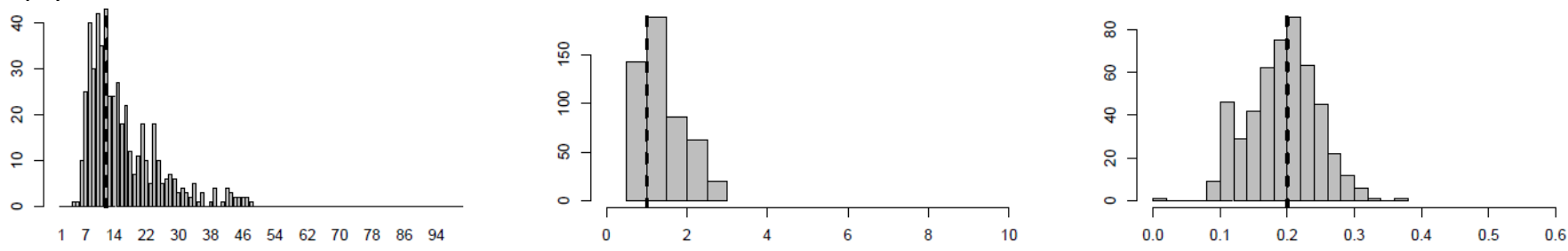

(b)
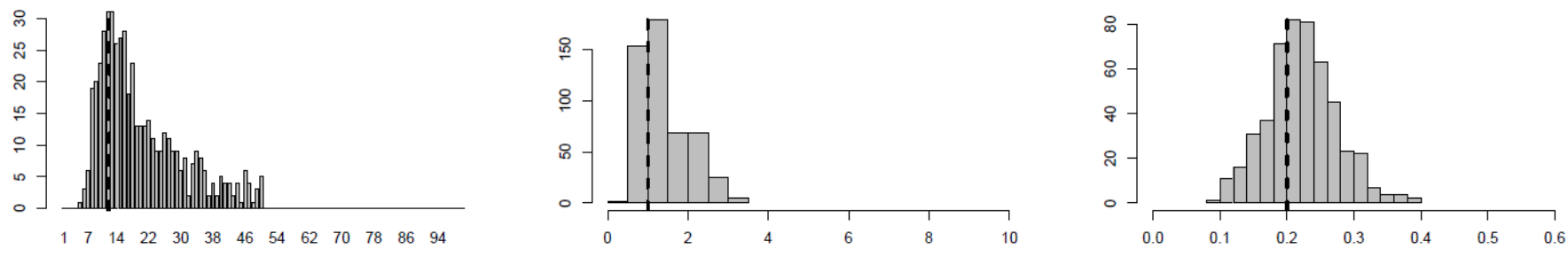

(c)
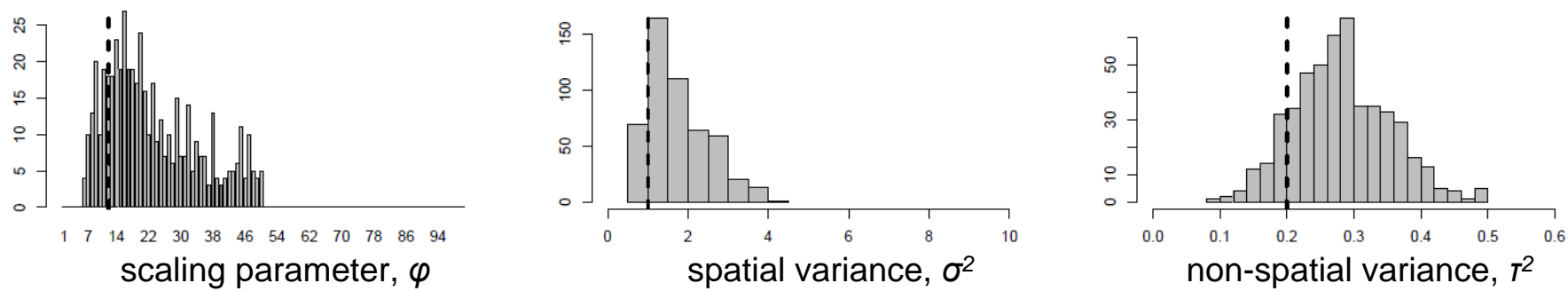


\section{Page 31 of 37}

(a)
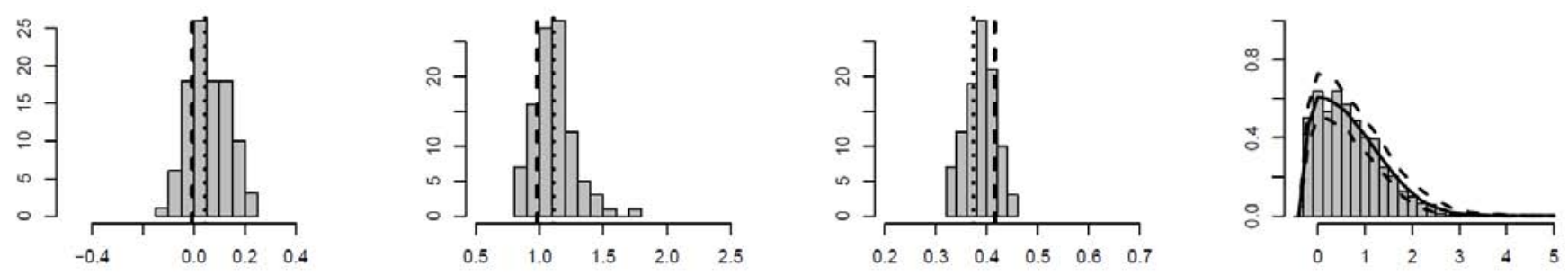

(b)
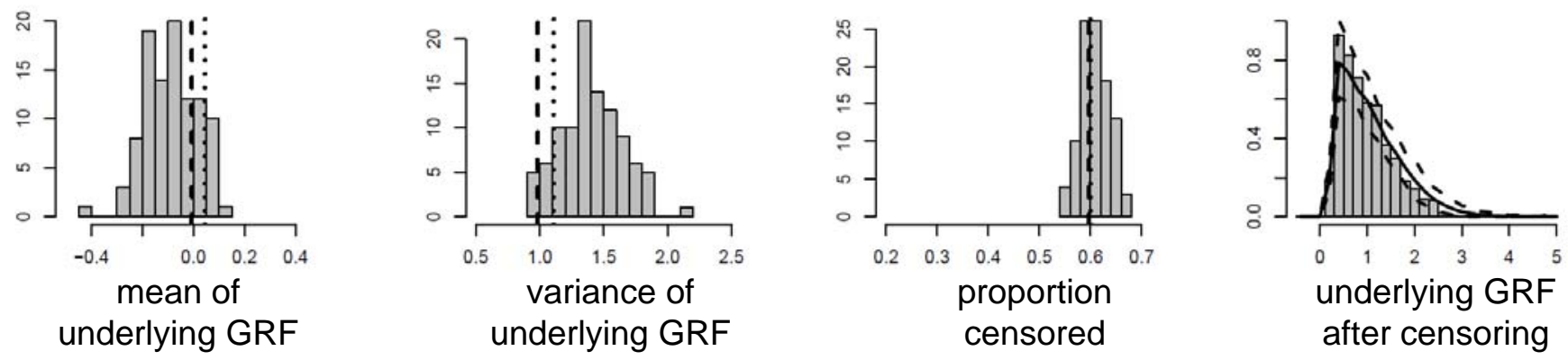
$\sim 40 \%$ of values censored
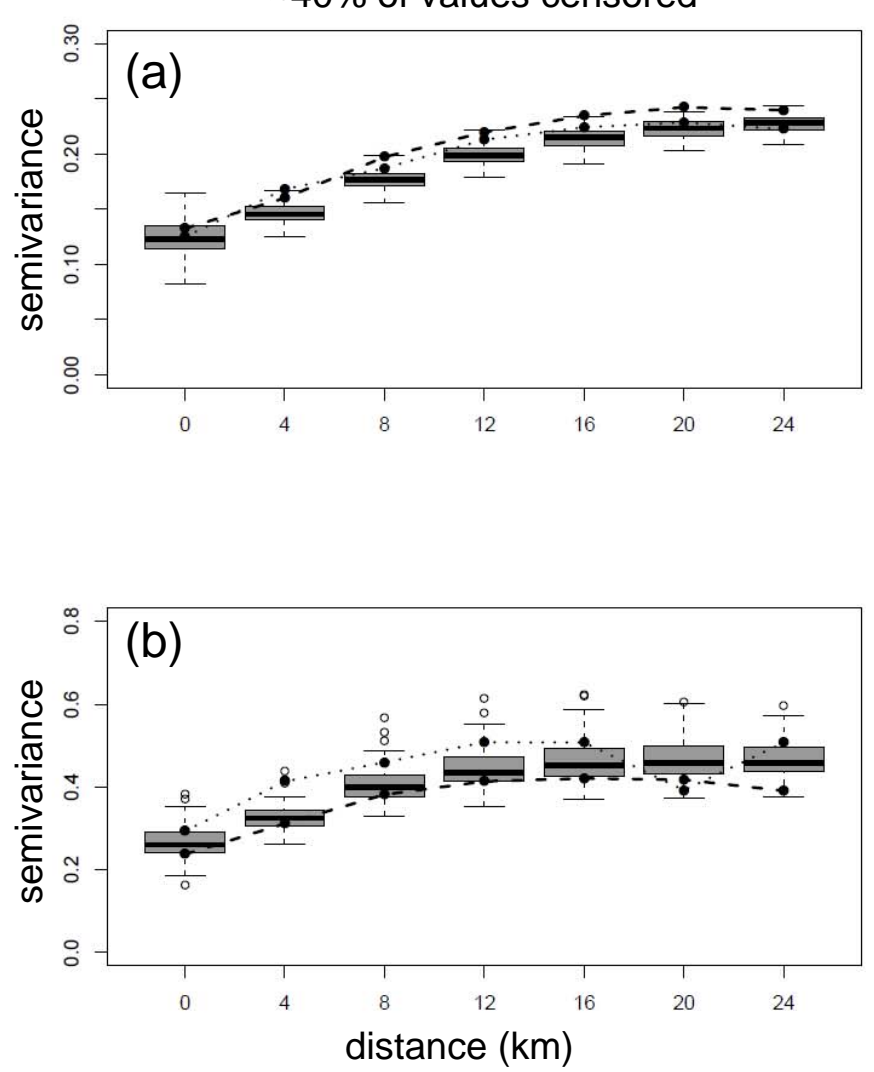

$-60 \%$ of values censored
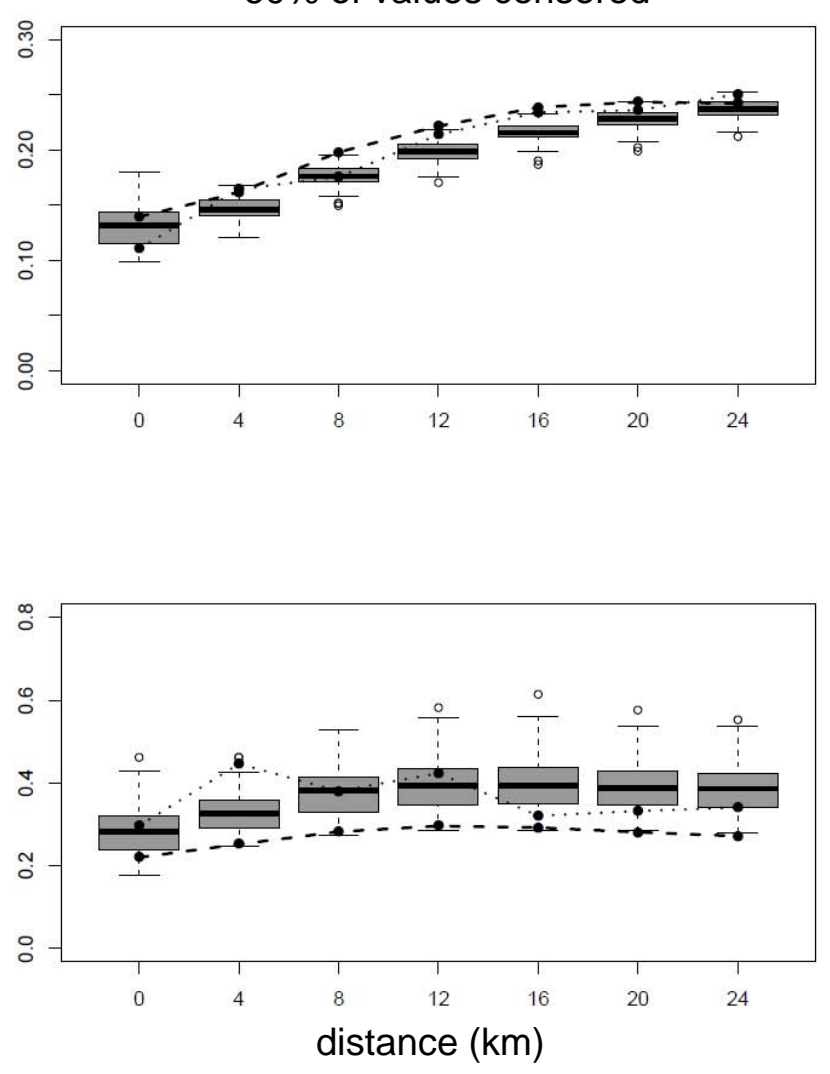
(a)

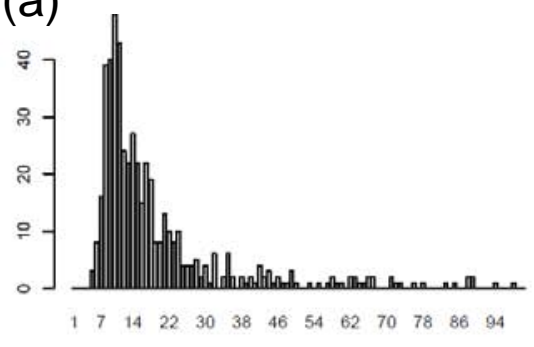

(b)

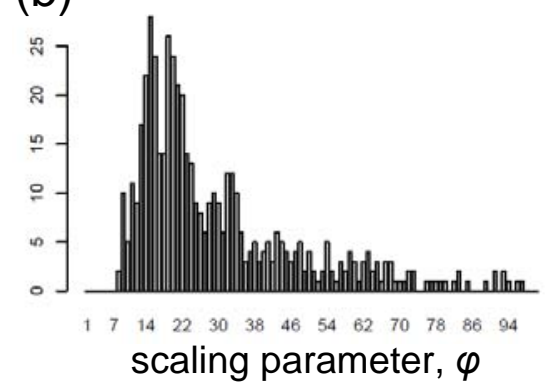

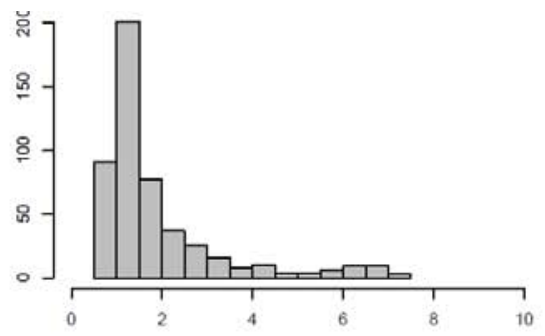
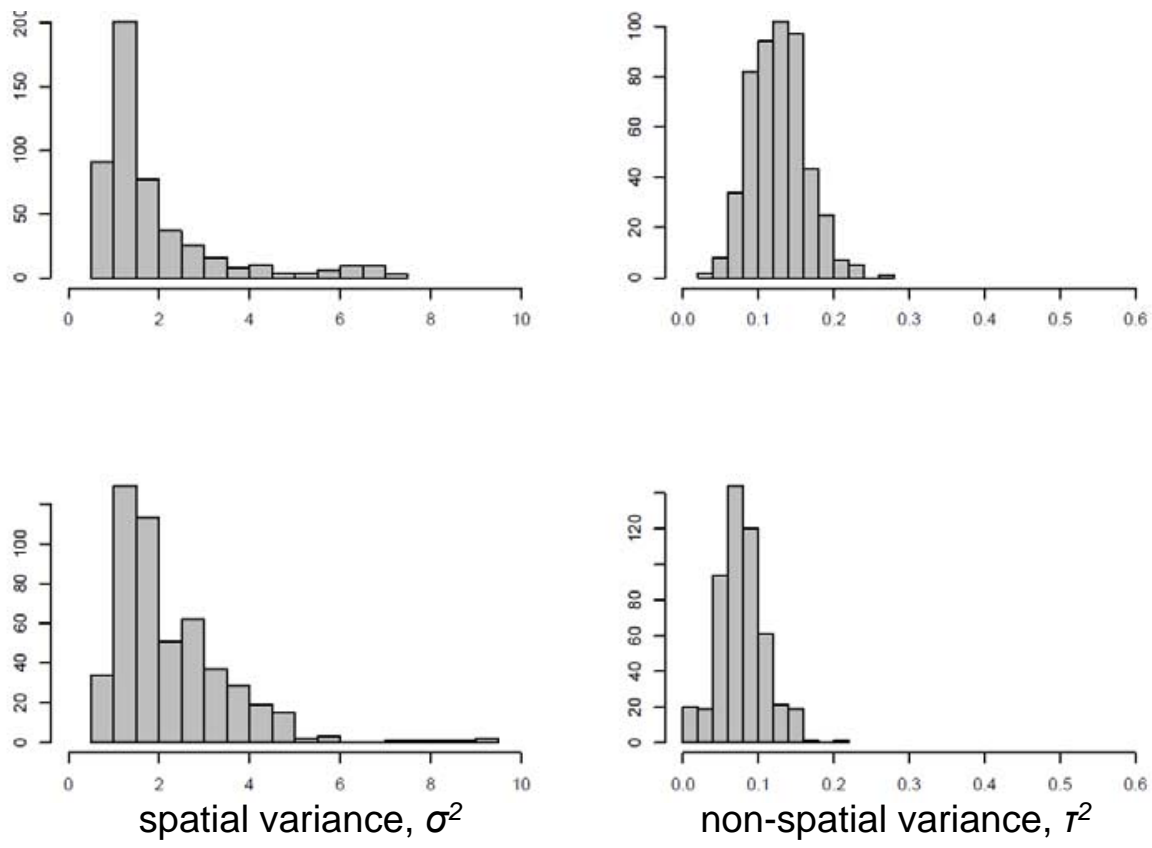


\section{Page 35 of 37}

(a)

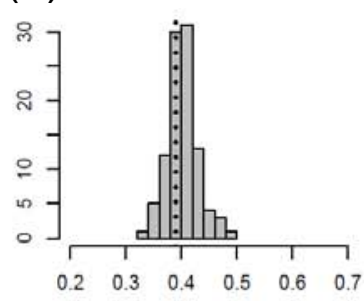

(b)

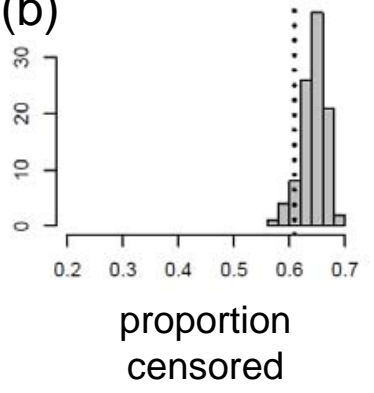

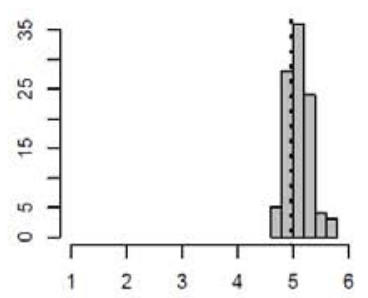

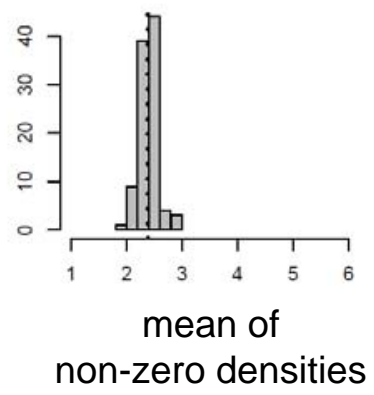

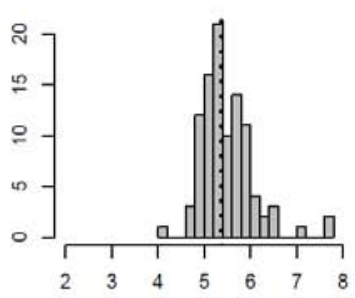

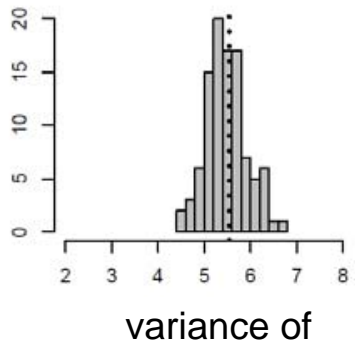
non-zero densities
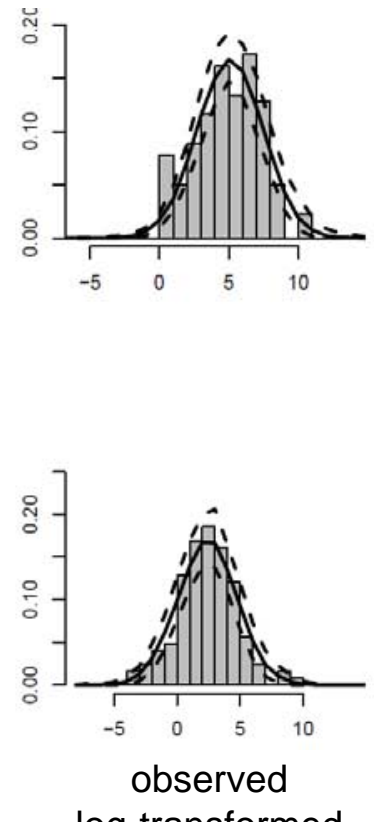
log-transformed non-zero densities 

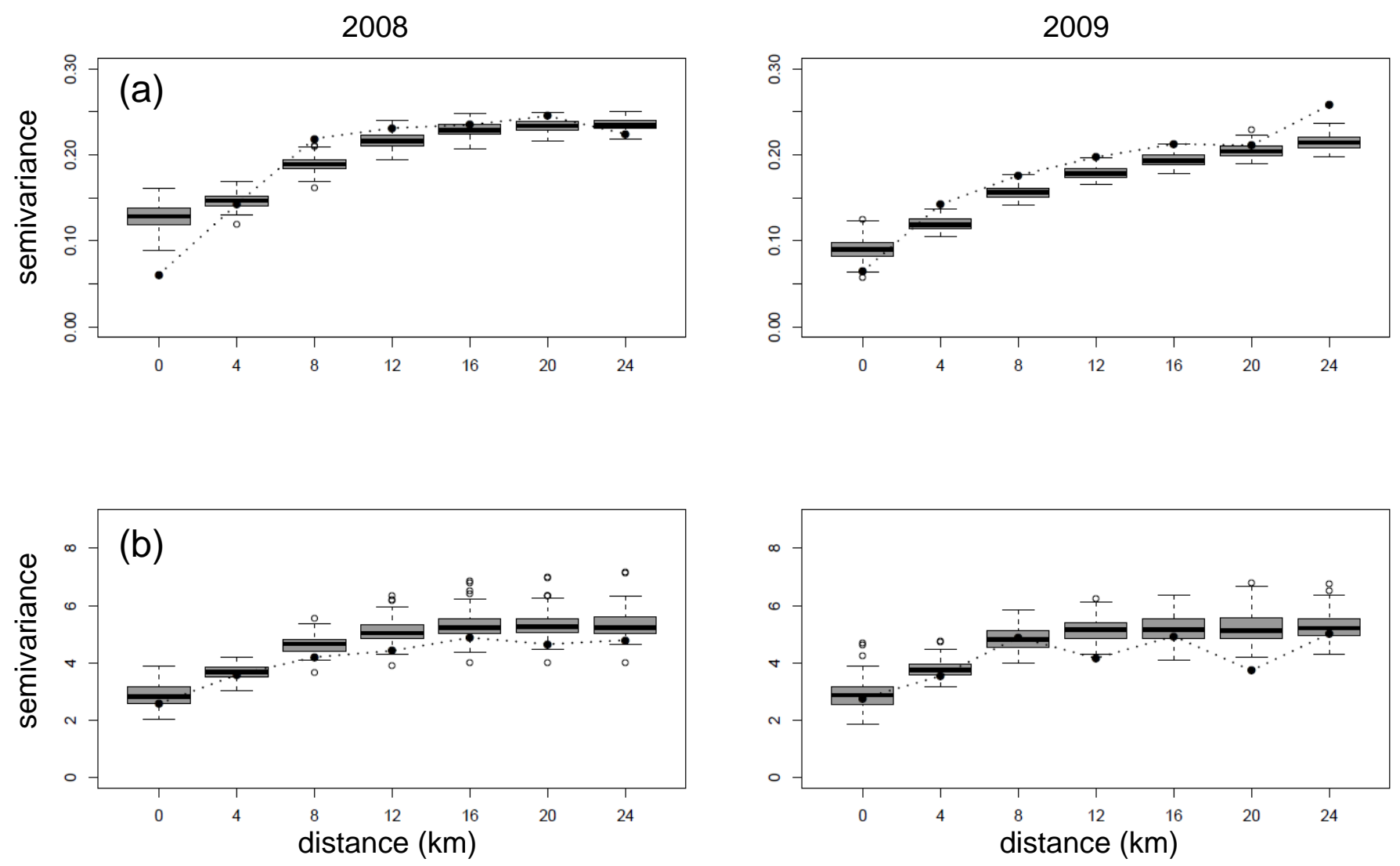
